\title{
THE REALIZATION PROBLEM FOR JØRGENSEN NUMBERS
}

\author{
YASUSHI YAMASHITA AND RYOSUKE YAMAZAKI
}

Abstract. Let $G$ be a two-generator subgroup of $\operatorname{PSL}(2, \mathbb{C})$. The Jørgensen number $J(G)$ of $G$ is defined by

$$
J(G)=\inf \left\{\left|\operatorname{tr}^{2} A-4\right|+|\operatorname{tr}[A, B]-2| ; G=\langle A, B\rangle\right\} .
$$

If $G$ is a non-elementary Kleinian group, then $J(G) \geq 1$. This inequality is called Jørgensen's inequality. In this paper, we show that, for any $r \geq 1$, there exists a non-elementary Kleinian group whose Jørgensen number is equal to $r$. This answers a question posed by Oichi and Sato. We also present our computer generated picture which estimates Jørgensen numbers from above in the diagonal slice of Schottky space.

\section{INTRODUCTION}

Let $G$ be a two-generator subgroup of $\operatorname{PSL}(2, \mathbb{C})$. Determining whether or not $G$ is discrete is an important problem in Kleinian group theory. In 1976, Jørgensen [7] showed that if $G=\langle X, Y\rangle$ is a non-elementary two-generator discrete subgroup of $\operatorname{PSL}(2, \mathbb{C})$ (Kleinian group), then

$$
\left|\operatorname{tr}^{2} X-4\right|+|\operatorname{tr}[X, Y]-2| \geq 1,
$$

where $[X, Y]=X Y X^{-1} Y^{-1}$. The Jørgensen number of an ordered pair $(X, Y)$ is defined as

$$
J(X, Y):=\left|\operatorname{tr}^{2} X-4\right|+|\operatorname{tr}[X, Y]-2|
$$

and the Jørgensen number of a non-elementary two-generator Kleinian group $G$ is defined as

$$
J(G):=\inf \{J(X, Y) \mid\langle X, Y\rangle=G\} .
$$

By (1), we have $J(G) \geq 1$. We can think of the Jørgensen number as measuring how far from being indiscrete. Jørgensen's inequality is sharp [7, and if $J(G)=1$, $G$ is called a Jørgensen group. There are many results on Jørgensen groups in the literature $[5,8,12,14,17,20,21,25]$. Also, it seems to be a difficult, but also challenging, problem to calculate Jørgensen numbers explicitly $3,6,22$. Oichi and Sato 18 asked the following natural question.

Question 1.1 (The realization problem). Let $r$ be a real number with $r \geq 1$. When is there a non-elementary Kleinian group whose Jorgensen number is equal to $r$ ?

Received by the editors August 21, 2017, and, in revised form, April 15, 2018, and September $26,2018$.

2010 Mathematics Subject Classification. Primary 30F40, 57M50.

Key words and phrases. Jørgensen's inequality, Jørgensen number, Kleinian groups.

This work was supported by JSPS KAKENHI Grant Number 26400088. 
Oichi and Sato [18] claimed that if $r=1,2,3$ or $r \geq 4$, then there is a nonelementary Kleinian group whose Jørgensen number is equal to $r$. (See also [3, 25].) Though Jørgensen's inequality is sharp, constructing a Kleinian group with small Jørgensen number (in particular, less than 4) is not easy. To the best of our knowledge, there is no known example of a classical Schottky group whose Jørgensen number is less than 4 . In this paper, we solve this realization problem.

Theorem 1.2. For any real number $r \geq 1$, there is a non-elementary two-generator discrete subgroup of $\operatorname{PSL}(2, \mathbb{C})$ whose Jørgensen number is $r$.

Jørgensen numbers can be studied by computer experiments. We will present a computer generated picture of the estimates of Jørgensen numbers from above in the diagonal slice of Schottky space. At the end of the paper, we present some Jørgensen groups, and we conjecture that they are counterexamples of Li-OichiSato's conjecture.

In section 2, we give a proof of Theorem 1.2 for the case $4 \leq r$. In section 3 , we give a proof for the case $1 \leq r \leq 4$. In section 4 , we present our computer generated picture of a one-parameter family of two-generator Kleinian groups, and describe our numerical calculation of the Jørgensen numbers of the groups in this family.

\section{THE PROOF FOR THE CASE $r \geq 4$}

Oichi and Sato 18 claimed that, for every real number $r \geq 4$, there is a subgroup $G$ of $\operatorname{PSL}(2, \mathbb{C})$ such that $J(G)=r$. But the proof was not written. For completeness, we give a short proof of this fact by calculating the Jørgensen numbers of the groups in the Riley slice.

First, we recall a classical result of Nielsen. A pair $\{X, Y\}$ of elements in the free group $F_{2}$ of rank two are called associated primitives if $X$ and $Y$ generate the group. For any associated primitives $\{A, B\}$ and $\{C, D\}$ of $F_{2}$, the commutator $[C, D]$ is conjugate to $[A, B]$ or $[A, B]^{-1}$. (See Theorem 3.9 in [15].) It follows that

Lemma 2.1. If $G$ is a subgroup of $\operatorname{PSL}(2, \mathbb{C})$ isomorphic to $F_{2}$, then the trace of the commutator of associated primitives of $G$ does not depend on the choice of associated primitives.

Let $\rho$ be a complex number, and define $G_{\rho}=\left\langle A, B_{\rho}\right\rangle$, where

$$
A=\left(\begin{array}{ll}
1 & 1 \\
0 & 1
\end{array}\right), \quad B_{\rho}=\left(\begin{array}{ll}
1 & 0 \\
\rho & 1
\end{array}\right) .
$$

Recall that the Riley slice is defined by

$$
\left\{\rho \in \mathbb{C} \mid G_{\rho} \text { is free and Kleinian and } \Omega\left(G_{\rho}\right) / G_{\rho} \text { is a } 4 \text {-times punctured sphere }\right\} \text {, }
$$

where $\Omega\left(G_{\rho}\right)$ is the regular set of $G_{\rho}$. (See [10,11] for the Riley slice.) If $\rho$ lies in the closure of the Riley slice, then $G_{\rho}$ is non-elementary, discrete, and free of rank two. Suppose that $G_{\rho}$ is isomorphic to $F_{2}$ and $\left\{A_{\rho}^{\prime}, B_{\rho}^{\prime}\right\}$ are any associated primitives for $G_{\rho}$. By Lemma 2.1, we have

$$
J\left(A_{\rho}^{\prime}, B_{\rho}^{\prime}\right)=\left|\operatorname{tr}^{2} A_{\rho}^{\prime}-4\right|+\left|\operatorname{tr}\left[A_{\rho}^{\prime}, B_{\rho}^{\prime}\right]-2\right| \geq\left|\operatorname{tr}\left[A, B_{\rho}\right]-2\right|=J\left(A, B_{\rho}\right)
$$

and $J\left(G_{\rho}\right)=J\left(A, B_{\rho}\right)=\left|\rho^{2}\right|$. Hence, the real numbers

$$
\left\{\left|\rho^{2}\right| \in \mathbb{R} \mid \rho \text { is in the closure of the Riley slice }\right\}
$$

are realized as Jørgensen numbers. In particular, since the closure of the Riley slice contains $\{y i \mid y \geq 2\}$, for any real $r \geq 4$, the Jørgensen number is realized. 


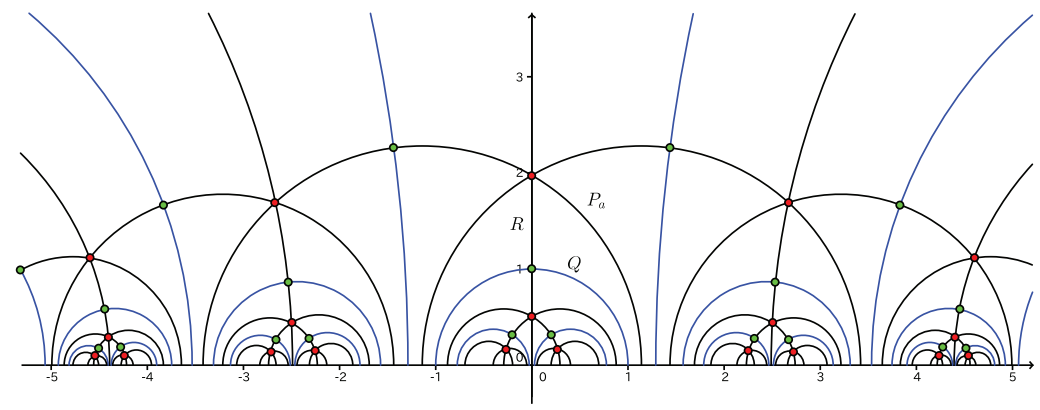

FIGURE 1. The axes of the generators and their orbits.

Remark 2.2. It is possible to improve the value 4 to around 2.467 by calculating the endpoints of the pleating rays of the Riley slice. See [26].

As an example of Kleinian groups that we can calculate the Jørgensen numbers by the same method, we consider the Maskit slice. Recall that, if $G$ is in the Maskit slice [9, 16, then it is discrete and isomorphic to $F_{2}$ and can be normalized as $G_{\mu}=\left\langle A, B_{\mu}\right\rangle$ for some complex number $\mu$, where

$$
A=\left(\begin{array}{ll}
1 & 2 \\
0 & 1
\end{array}\right), B_{\mu}=\left(\begin{array}{cc}
-i \mu & -i \\
-i & 0
\end{array}\right)
$$

Since $\left|\operatorname{tr}^{2} A-4\right|=0$, by the same argument for the Riley slice, we have $J\left(G_{\mu}\right)=$ $J\left(A, B_{\mu}\right)=4$.

\section{The PRoof FOR THE CASE $1 \leq r \leq 4$}

In this section, we give a proof for the case $1 \leq r \leq 4$. For simplicity, we use the same notation for a matrix in $\operatorname{SL}(2, \mathbb{C})$ and its equivalence class in $\operatorname{PSL}(2, \mathbb{C})$.

3.1. The basic configuration. Let $z, z^{\prime} \in \mathbb{C}$, and let $\left[z, z^{\prime}\right]$ be the oriented line from $z$ to $z^{\prime}$ in $\mathbb{H}^{3}$. Following [4, we define the line matrix associated with $\left[z, z^{\prime}\right]$ as

$$
M\left(\left[z, z^{\prime}\right]\right):=\frac{i}{z^{\prime}-z}\left(\begin{array}{cc}
z+z^{\prime} & -2 z z^{\prime} \\
2 & -z-z^{\prime}
\end{array}\right) .
$$

See [4, p. 64, equation (1). It is the order two rotation about $\left[z, z^{\prime}\right]$.

For $a \geq 1$, set

$$
P_{a}:=M([a,-3 a]), \quad Q:=M([1,-1]), \quad R:=M([0, \infty])=\left(\begin{array}{cc}
i & 0 \\
0 & -i
\end{array}\right) .
$$

Let $G_{a}$ be a subgroup of $\operatorname{PSL}(2, \mathbb{C})$ generated by $P_{a}, Q$, and $R$. The axes of the generators are contained in the vertical plane $\{(x, 0, z) \mid z>0\} \cong \mathbb{H}^{2}$. Since we assumed that $a \geq 1,[a,-3 a]$ and $[1,-1]$ do not intersect in $\mathbb{H}^{3}$. The angle between $[1,-1]$ and $[0, \infty]$ is $\pi / 2$, and the angle between $[0, \infty]$ and $[a,-3 a]$ is $\pi / 3$. See Figure 1. Hence, as an abstract group, $G_{a}$ has a presentation

$$
\left\langle P_{a}, Q, R \mid P_{a}^{2}=Q^{2}=R^{2}=(Q R)^{2}=\left(R P_{a}\right)^{3}=i d\right\rangle
$$

for any $a \geq 1$ and is isomorphic to a hyperbolic (full) triangle group in $\mathbb{H}^{2}$. (Note that hyperbolic (full) triangle groups are defined as groups generated by reflections, but $G_{a}$ is generated by rotations in $\mathbb{H}^{3}$.) Hence, $G_{a}$ is non-elementary and discrete. 
Remark 3.1. Groups conjugate to $\left\langle P_{a}, Q, R\right\rangle$ were studied by Series, Tan, and the first author in 23 .

For later purpose, we classify non-trivial elements of $G_{a}$ into three types:

(i) elements conjugate to $P_{a}, Q, R, Q R$, or $R P_{a}$,

(ii) elements conjugate to $\left(P_{a} Q\right)^{n}$ for some $n \in \mathbb{Z} \backslash\{0\}$,

(iii) others.

First, we consider the case $a=1$.

Lemma 3.2. Let $E_{1}$ be a non-trivial element in $G_{1}$. Then

(i) $E_{1}$ is of type (i) if and only if $\operatorname{tr} E_{1} \in\{0, \pm 1\}$.

(ii) $E_{1}$ is of type (ii) if and only if $\operatorname{tr} E_{1}= \pm 2$.

(iii) $E_{1}$ is of type (iii) if and only if $\operatorname{tr} E_{1} \in\{ \pm 3, \pm 4, \ldots\} \cup\{ \pm i, \pm 2 i, \ldots\}$.

Proof. By construction, if $E_{1}$ is of type (i) (resp., (ii)), then $\operatorname{tr} E_{1} \in\{0, \pm 1\}$ (resp., $\operatorname{tr} E_{1}= \pm 2$ ).

For the converse, set

$$
M=\left(\begin{array}{cc}
\frac{1}{\sqrt{2}} & \frac{1}{\sqrt{2}} \\
0 & \sqrt{2}
\end{array}\right)
$$

Then, we have

$$
M P_{1} M^{-1}=\left(\begin{array}{cc}
0 & -i \\
-i & 0
\end{array}\right), M Q M^{-1}=\left(\begin{array}{cc}
-i & 0 \\
-2 i & i
\end{array}\right), M R M^{-1}=\left(\begin{array}{cc}
i & -i \\
0 & -i
\end{array}\right) .
$$

Hence, for any element $E_{1} \in G_{1}$, we have

$$
\operatorname{tr} E_{1} \in\{0, \pm 1, \pm 2, \pm 3, \ldots\} \cup\{ \pm i, \pm 2 i, \pm 3 i, \ldots\} .
$$

Note that $G_{1}$ acts on $\{(x, 0, z) \mid z>0\} \cong \mathbb{H}^{2}$ as the hyperbolic (full) triangle group of type $(2,3, \infty)$ (extended modular group). Let $F_{1}$ be the (ideal) triangle of type $(2,3, \infty)$ bounded by the axes of $P_{1}, Q$, and $R$. Then, $F_{1}$ is a fundamental region for the action of $G_{1}$. For each point in the interior of $F_{1}$, the stabilizer is trivial. For each point in the interior of the edges, the stabilizer is $\left\langle P_{1}\right\rangle,\langle Q\rangle$, or $\langle R\rangle$. For the (non-ideal) vertices, the stabilizers are $\langle Q, R\rangle$ and $\left\langle R, P_{1}\right\rangle$. For the ideal vertex, the stabilizer is $\left\langle P_{1}, Q\right\rangle$.

If $\operatorname{tr} E_{1} \in\{0, \pm 1\}$, then $E_{1}$ is elliptic and has a fixed point in $\mathbb{H}^{2}$, and this point has non-trivial stabilizer. The observation in the previous paragraph shows that $E_{1}$ is of type (i). If $\operatorname{tr} E_{1}= \pm 2$, then $E_{1}$ is parabolic and has a parabolic fixed point on $\partial \mathbb{H}^{2}$. The observation in the previous paragraph shows that $E_{1}$ is of type (ii).

Since we have shown the equivalences in (i) and (ii), the equivalence in (iii) follows from (2).

Remark 3.3. By this proof, we see that $G_{1}$ is a conjugate to a subgroup of the Picard group. Sato 22] showed that the Picard group is a Jorgensen group. GonzálezAcuña and Ramírez [6] described all Jørgensen subgroups of the Picard group.

Since the abstract group structure will not change for any $a \geq 1$, essentially the same type of arguments as in the proof of the previous lemma shows the following.

Lemma 3.4. Let $E_{a}$ be a non-trivial element in $G_{a}$ for $a \geq 1$. Then $E_{a}$ is of type (i) if and only if $\operatorname{tr} E_{a} \in\{0, \pm 1\}$. 
Next, we consider type (iii) for $a \geq 1$. First, we prove a technical lemma. Let $a^{\prime}$ be a number with $1 \leq a^{\prime}<a$. Let $F_{a}$ (resp., $F_{a^{\prime}}$ ) be the region bounded by the axes of $P_{a}$ (resp., $\left.P_{a^{\prime}}\right), Q$, and $R$. We define a map $f$ from $\left(\partial F_{a}\right) \cap \mathbb{H}^{2}$ to $\left(\partial F_{a^{\prime}}\right) \cap \mathbb{H}^{2}$ as follows:

$$
f(p)= \begin{cases}\frac{a^{\prime}}{a} \cdot p & \left(\text { if }|p|>\frac{a}{a^{\prime}}\right), \\ \frac{p}{|p|} & \left(\text { if }|p| \leq \frac{a}{a^{\prime}}\right) .\end{cases}
$$

We denote the hyperbolic distance in $\mathbb{H}^{2}$ by $d$.

Lemma 3.5. For any $p, p^{\prime} \in\left(\partial F_{a}\right) \cap \mathbb{H}^{2}$, we have $d\left(f(p), f\left(p^{\prime}\right)\right) \leq d\left(p, p^{\prime}\right)$.

Proof. Set $q=f(p), q^{\prime}=f\left(p^{\prime}\right)$. We denote the axes of $P_{a}, Q$, and $R$ by $A\left(P_{a}\right), A(Q)$, and $A(R)$, respectively.

Suppose that $p \in A\left(P_{a}\right)$ and $p^{\prime} \in A(R)$. We have $|p|>a>\frac{a}{a^{\prime}}$. If $\left|p^{\prime}\right|>\frac{a}{a^{\prime}}$, then we have

$$
d\left(f(p), f\left(p^{\prime}\right)\right)=d\left(\frac{a^{\prime}}{a} \cdot p, \frac{a^{\prime}}{a} \cdot p^{\prime}\right)=d\left(p, p^{\prime}\right) .
$$

Thus, it suffices to consider the case $\left|p^{\prime}\right| \leq \frac{a}{a^{\prime}}$. To compare $d\left(p, p^{\prime}\right)$ and $d\left(q, q^{\prime}\right)$, we consider two hyperbolic triangles given by $p, p^{\prime}, p^{\prime \prime}$ and $q, q^{\prime}, q^{\prime \prime}$, where $p^{\prime \prime}$ (resp., $q^{\prime \prime}$ ) is the intersection of the axes of $P_{a}$ (resp., $P_{a^{\prime}}$ ) and $R$. That is,

$$
p^{\prime \prime}=\sqrt{3} a \cdot i, \quad q^{\prime \prime}=\sqrt{3} a^{\prime} \cdot i \quad(i=\sqrt{-1}) .
$$

By the hyperbolic low of cosines,

$$
\cosh d\left(p, p^{\prime}\right)=g\left(d\left(p, p^{\prime \prime}\right), d\left(p^{\prime}, p^{\prime \prime}\right)\right), \quad \cosh d\left(q, q^{\prime}\right)=g\left(d\left(q, q^{\prime \prime}\right), d\left(q^{\prime}, q^{\prime \prime}\right)\right),
$$

where

$$
g(x, y)=\cosh x \cdot \cosh y-\sinh x \cdot \sinh y \cdot \frac{1}{2} .
$$

(Recall that the angle between the axes of $P_{a}$ (or $P_{a^{\prime}}$ ) and $R$ is $\pi / 3$, so its cosine is $\frac{1}{2}$.) Since $d\left(q, q^{\prime \prime}\right)=d\left(p, p^{\prime \prime}\right)$ and $d\left(q^{\prime}, q^{\prime \prime}\right)<d\left(p^{\prime}, p^{\prime \prime}\right)$, if we can show that $g(x, y)$ is monotonically increasing in $y$ for each fixed $x$, we have $d\left(q, q^{\prime}\right)<d\left(p, p^{\prime}\right)$. Since we have

$$
\begin{aligned}
\frac{d}{d y} g(x, y) & =\cosh x \cdot \sinh y-\sinh x \cdot \cosh y \cdot \frac{1}{2} \\
& >\cosh x \cdot \sinh y-\cosh x \cdot \cosh y \cdot \frac{1}{2} \\
& =\cosh x\left(\sinh y-\cosh y \cdot \frac{1}{2}\right),
\end{aligned}
$$

and $\cosh x$ is always positive and the second factor is positive if $y>\frac{\log 3}{2}$, then $g(x, y)$ is monotonically increasing in $y$ if $y>\frac{\log 3}{2}$. Our conditions $\left|p^{\prime}\right|<\frac{a}{a^{\prime}}$ and $1<a^{\prime}$ imply that

$$
\begin{aligned}
& d\left(p^{\prime}, p^{\prime \prime}\right)=\log \frac{\left|p^{\prime \prime}\right|}{\left|p^{\prime}\right|}>\log \frac{\sqrt{3} a}{\frac{a}{a^{\prime}}}>\frac{\log 3}{2} \\
& d\left(q^{\prime}, q^{\prime \prime}\right)=\log \frac{\left|q^{\prime \prime}\right|}{\left|q^{\prime}\right|}=\log \frac{\sqrt{3} a^{\prime}}{1}>\frac{\log 3}{2} .
\end{aligned}
$$

Hence, $d\left(q, q^{\prime}\right)<d\left(p, p^{\prime}\right)$, as required. 


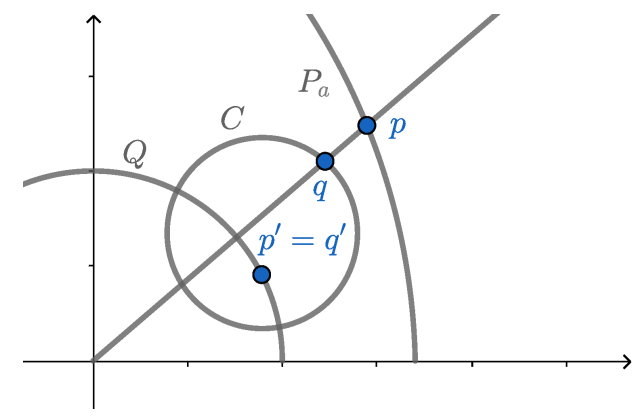

Figure 2. The case $p \in A\left(P_{a}\right)$ and $p^{\prime} \in A(Q)$.

Next, suppose that $p \in A\left(P_{a}\right)$ and $p^{\prime} \in A(Q)$. As in the previous case, $q=\frac{a^{\prime}}{a} \cdot p$. Since $p^{\prime} \in A(Q)$ is on the unit circle, $q^{\prime}=f\left(p^{\prime}\right)=\frac{p^{\prime}}{\left|p^{\prime}\right|}=p^{\prime}$, and $q^{\prime}$ is on $A(Q)$. Let $C$ be the hyperbolic circle with center $q^{\prime}$ that passes through $q . C$ is a Euclidean circle and orthogonal to $A(Q)$. See Figure 2, Hence, any Euclidean line passing through the origin meets $C$ once inside $A(Q)$ and once outside $A(Q)$. Thus, we see that the point $p$ is outside of $C$, and $d\left(q, q^{\prime}\right)<d\left(p, p^{\prime}\right)$.

Other cases $\left(\left(p, p^{\prime}\right) \in A(Q) \times A(R), A\left(P_{a}\right) \times A\left(P_{a}\right), A(Q) \times A(Q), A(R) \times A(R)\right)$ are easy and omitted.

Lemma 3.6. Let $E_{a}$ be an element of type (iii) in $G_{a}$. Then, we have

$$
\operatorname{tr} E_{a} \in\{ \pm x \mid x \in \mathbb{R}, x \geq 3\} \cup\{ \pm y i \mid y \in \mathbb{R}, y \geq 1\} .
$$

Proof. First, we show that $\left|\operatorname{tr} E_{a}\right|$ is (weakly) increasing in $a$. Let $a^{\prime}$ be a number with $1 \leq a^{\prime} \leq a$, and suppose that $E_{a}$ and $E_{a^{\prime}}$ are loxodromic. Since $E_{a}$ preserves the vertical plane $\mathbb{H}^{2}$ in $\mathbb{H}^{3}, E_{a}$ or $E_{a}^{-1}$ is conjugate to

$$
\left(\begin{array}{cc}
\sqrt{l_{a}} & 0 \\
0 & 1 / \sqrt{l_{a}}
\end{array}\right) \text { or }\left(\begin{array}{cc}
\sqrt{l_{a}} i & 0 \\
0 & 1 / \sqrt{l_{a}} i
\end{array}\right)
$$

where $l_{a}$ is the translation length of $E_{a}$. Thus, we have

$$
\operatorname{tr} E_{a}= \pm\left(\sqrt{l_{a}}+1 / \sqrt{l_{a}}\right) \text { or } \pm\left(\sqrt{l_{a}}-1 / \sqrt{l_{a}}\right) i .
$$

We claim that $l_{a}$ is (weakly) increasing in $a$.

To see this, let $m_{a} \in \mathbb{H}^{2}$ be the axis of $E_{a}$. Since $F_{a}$ is a fundamental region for $G_{a}$ in $\mathbb{H}^{2}$, we can map $m_{a}$ into $F_{a}$ by the elements in $G_{a}$. The image is a closed trajectory of the hyperbolic billiard of billiard table $F_{a}$. Let $p_{1}, \ldots, p_{n}$ be the points on the trajectory where it hits the sides (i.e., the boundary) of the table $F_{a}$ so that

$$
l_{a}=\sum_{i=1}^{n} d\left(p_{i}, p_{i+1}\right) .
$$

Here, the indices are understood $\bmod n$. See Figure 3 . Let $f$ be the function defined before Lemma 3.5. and put $q_{i}=f\left(p_{i}\right)$ for $i=1, \ldots, n$. By Lemma 3.5. $d\left(q_{i}, q_{i+1}\right) \leq d\left(p_{i}, p_{i+1}\right)$ for $i=1, \ldots, n$.

Let $t$ be the closed piecewise geodesic given by connecting $q_{1}, q_{2}, \ldots, q_{n}, q_{1}$. Let $\widehat{t}$ be the "unfolding" of $t$ starting from a segment in $t$ connecting, say, $q_{1}$ and $q_{2}$. See Figure 4, $\widehat{t}$ is a bi-infinite piecewise geodesic in $\mathbb{H}^{2}$. Let . . , $\widehat{q_{-2}}, \widehat{q_{-1}}, \widehat{q_{0}}, \widehat{q_{1}}, \widehat{q_{2}}, \widehat{q_{3}}, \ldots$ be the vertices of $\widehat{t}$ with $\widehat{q_{1}}=q_{1}$ and $\widehat{q_{2}}=q_{2}$. The vertex $\widehat{q_{i}}$ in $\widehat{t}$ is mapped to 


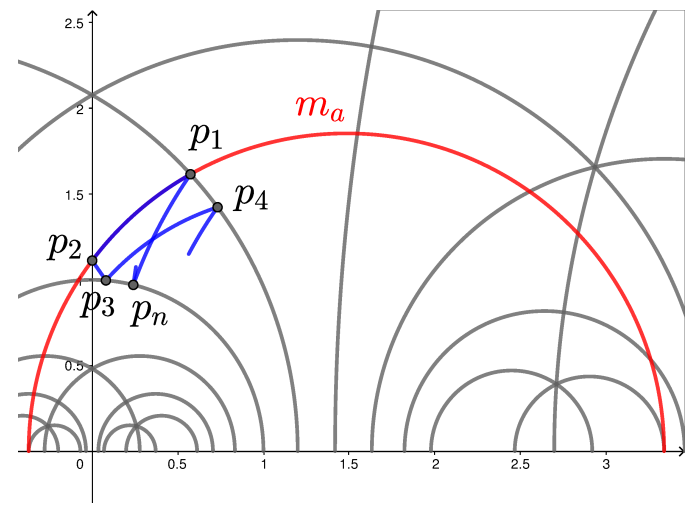

Figure 3. The axis $m_{a}$ of a type (iii) element and (a part of) the hyperbolic billiard.

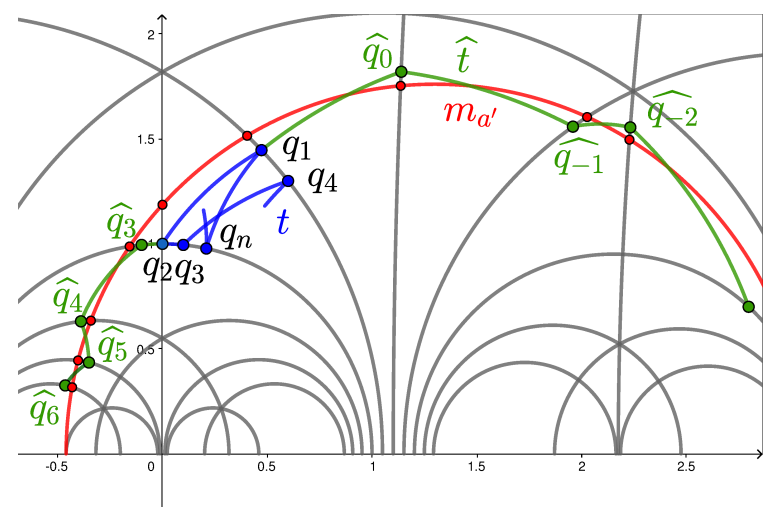

Figure 4. The unfolding $\widehat{t}$ of $t$ and the axis $m_{a^{\prime}}$.

$q_{j}$ in $t$ with $i \equiv j(\bmod n)$ by the action of $G_{a^{\prime}}$. In particular, we have either $E_{a^{\prime}}\left(\widehat{q_{1}}\right)=\widehat{q_{n+1}}$ or $E_{a^{\prime}}\left(\widehat{q_{n+1}}\right)=\widehat{q_{1}}$ depending on the choice of numbering. Thus, we see that

$$
l_{a^{\prime}} \leq d\left(\widehat{q_{1}}, \widehat{q_{n+1}}\right) \leq \sum_{i=1}^{n} d\left(\widehat{q_{i}}, \widehat{q_{i+1}}\right)=\sum_{i=1}^{n} d\left(q_{i}, q_{i+1}\right) .
$$

Thus, we have

$$
l_{a^{\prime}} \leq \sum_{i=1}^{n} d\left(q_{i}, q_{i+1}\right) \leq \sum_{i=1}^{n} d\left(p_{i}, p_{i+1}\right)=l_{a},
$$

as required. Hence, by formula (3), we have $\left|\operatorname{tr} E_{a^{\prime}}\right| \leq\left|\operatorname{tr} E_{a}\right|$.

Since all the entries of $P_{a}, Q$, and $R$ are pure imaginary numbers, $\operatorname{tr} E_{a}$ is always real if the word length of $E_{a}$ is even, and pure imaginary if it is odd. By Lemma 3.2 (iii) and the arguments in the previous paragraphs, tr $E_{a}$ cannot be in $\{ \pm x \mid x \in$ $\mathbb{R}, 2<x<3\} \cup\{ \pm y i \mid y \in \mathbb{R}, 0<y<1\}$. (Recall that, at the beginning of the first paragraph of this proof, we assumed that $E_{a}$ is loxodromic. For $a^{\prime}$, we can take $a^{\prime}=1$.) Since $\operatorname{tr} E_{a}$ is continuous on $a, E_{a}$ cannot be in $\{ \pm x \mid x \in \mathbb{R}, 0 \leq x \leq 2\}$, too. 
3.2. A generating pair. We define

$$
A_{a}:=P_{a} Q=\left(\begin{array}{cc}
-3 a / 2 & 1 / 2 \\
-1 / 2 & -1 / 2 a
\end{array}\right), \quad B:=R=\left(\begin{array}{cc}
i & 0 \\
0 & -i
\end{array}\right) .
$$

Since $Q=A_{a} B A_{a}^{-1} B^{-1} A_{a} B,\left(A_{a}, B\right)$ is a generating pair of $G_{a}$, and $G_{a}$ is a two-generator group. As an abstract group, by Tietze transformation, we have

$$
\begin{aligned}
G_{a} & =\left\langle P_{a}, Q, B, A_{a} \mid P_{a}^{2}=Q^{2}=B^{2}=(Q B)^{2}=\left(B P_{a}\right)^{3}=i d, A_{a}=P_{a} Q\right\rangle \\
& =\left\langle A_{a}, B \mid \begin{array}{l}
\left(A_{a} B A_{a}^{-1} B A_{a} B A_{a}^{-1}\right)^{2}=\left(A_{a} B A_{a}^{-1} B^{-1} A_{a} B\right)^{2}=B^{2} \\
=\left(A_{a} B A_{a}^{-1} B^{-1} A_{a} B \cdot B\right)^{2}=\left(B \cdot A_{a} B A_{a}^{-1} B A_{a} B A_{a}^{-1}\right)^{3}=i d
\end{array}\right\rangle \\
(4) & =\left\langle A_{a}, B \mid\left(A_{a} B A_{a}^{-1} B^{-1} A_{a} B\right)^{2}=B^{2}=\left(A_{a} B A_{a}^{-1} B^{-1} A_{a}\right)^{2}=i d\right\rangle .
\end{aligned}
$$

Remark 3.7. A conjugate to this generating pair $A_{a}$ and $B$ was studied in [23] and called the singular solid torus.

Lemma 3.8. In $G_{a}$, any element of order three cannot be a part of a minimal generating system.

Proof. Let $\tau$ be the map from the free group $F_{2}=\langle\alpha, \beta\rangle$ of rank two to $\{0,1\}^{2}$ that counts the exponents of $\alpha$ and $\beta \bmod 2$ in any word of $F_{2}$. Since

$$
\tau\left(\left(\alpha \beta \alpha^{-1} \beta^{-1} \alpha \beta\right)^{2}\right)=\tau\left(\beta^{2}\right)=\tau\left(\left(\alpha \beta \alpha^{-1} \beta^{-1} \alpha\right)^{2}\right)=(0,0),
$$

$\tau$ is well-defined on $G_{a}$. We denote the set $\left\{X \in G_{a} \mid \tau(X)=(i, j)\right\}$ by $G_{(i, j)}$. $G_{(i, j)}$ is not an empty set for $(i, j)=(0,0),(0,1),(1,0),(1,1)$.

Let $X$ be an element of $G$ such that $X^{3}=i d$. Then, $\tau(X)=(0,0)$. (Otherwise, we have $\tau\left(X^{3}\right) \equiv \tau\left(X^{2}\right)+\tau(X) \equiv \tau(X) \not \equiv(0,0)=\tau(i d)(\bmod 2)$, which is a contradiction.) Hence, for any $Y \in G_{a}$, we have

$$
\langle X, Y\rangle \subset G_{(0,0)} \cup G_{\tau(Y)} \neq G_{(0,0)} \cup G_{(1,0)} \cup G_{(0,1)} \cup G_{(1,1)}=G_{a}
$$

and the lemma is proved.

3.3. The Jørgensen number. Finally, we calculate the Jørgensen number.

Proposition 3.9. For any $1 \leq a \leq a_{0}$, where $a_{0}=(\sqrt{7}+2) / 3$, we have

$$
J\left(G_{a}\right)=J\left(A_{a}, B\right)=\frac{\left(3 a^{2}-1\right)^{2}}{4 a^{2}} .
$$

In particular, $J\left(G_{1}\right)=1$ and $J\left(G_{a_{0}}\right)=4$. For any $1 \leq r \leq 4$, the Jørgensen number is realized.

Proof. Since $\operatorname{tr}^{2} A_{a}=\left(3 a^{2}+1\right)^{2} / 4 a^{2}$ and $\operatorname{tr}\left[A_{a}, B\right]=1$, for $a \geq 1$, we have

$$
J\left(A_{a}, B\right)=\left|\frac{\left(3 a^{2}+1\right)^{2}}{4 a^{2}}-4\right|+1=\frac{\left(3 a^{2}-1\right)^{2}}{4 a^{2}} .
$$

Since $a_{0}$ satisfies the equation $\left(3 a_{0}^{2}-1\right)^{2} / 4 a_{0}^{2}=4$, if $1 \leq a \leq a_{0}$, we have $1 \leq$ $J\left(A_{a}, B\right) \leq 4$. To prove this proposition by contradiction, suppose that there exist $C, D \in G_{a}$ such that $J(C, D)<J\left(A_{a}, B\right)$ and $G_{a}=\langle C, D\rangle$. Since $G_{a}$ is non-elementary, $|\operatorname{tr}[C, D]-2|>0$. (See p. 68 in [1].) 
Case 1: $C$ is of type (i). If $\operatorname{tr} C=0$, then

$$
J(C, D)=\left|0^{2}-4\right|+|\operatorname{tr}[C, D]-2|>4 \geq J\left(A_{a}, B\right),
$$

and this cannot happen. If $\operatorname{tr} C= \pm 1$, then $C$ is an elliptic element of order three. Since $C$ cannot be a part of a minimal generating system by Lemma 3.8, this cannot happen.

Case 2: $C$ is of type (ii). $C$ can be written as $K\left(P_{a} Q\right)^{n} K^{-1}$ for some $K \in G_{a}$ and $n \neq 0$.

Case 2-1: $|n|>1$. We claim that $G_{a} \neq\langle C, D\rangle$. To see this, consider the Coxeter group

$$
H_{n}:=\left\langle P, Q, R \mid P^{2}=Q^{2}=R^{2}=(P R)^{3}=(Q R)^{2}=(P Q)^{n}=i d\right\rangle
$$

and let $\pi: G_{a} \rightarrow H_{n}$ be the natural surjection. Since $\pi(C)=\pi\left(K\left(P_{a} Q\right)^{n} K^{-1}\right)=$ $i d$, we have

$$
\pi(\langle C, D\rangle)=\langle\pi(D)\rangle \neq H_{n},
$$

because $H_{n}$ is not cyclic when $|n|>1$. It follows that $G_{a} \neq\langle C, D\rangle$ and the claim is proved.

Case 2-2: $|n|=1$. In this case, $\operatorname{tr}^{2} C=\operatorname{tr}^{2} A_{a}$.

Case 2-2-1: $[C, D]$ is of type (i). If $\operatorname{tr}[C, D]=0$, then

$$
J(C, D)=\left|\operatorname{tr}^{2} A_{a}-4\right|+|0-2|>\left|\operatorname{tr}^{2} A_{a}-4\right|+|1-2|=J\left(A_{a}, B\right) .
$$

If $\operatorname{tr}[C, D]= \pm 1$,

$$
J(C, D)=\left|\operatorname{tr}^{2} C-4\right|+|\operatorname{tr}[C, D]-2|=\left|\operatorname{tr}^{2} A_{a}-4\right|+| \pm 1-2| \geq J\left(A_{a}, B\right) .
$$

Case 2-2-2: $[C, D]$ is of type (ii). In this case, $\operatorname{tr}[C, D]= \pm \operatorname{tr} A_{a}^{m}$ for some $m \neq 0$. Since $\operatorname{tr} A_{a}= \pm(-3 a / 2-1 / 2 a), A_{a}$ is hyperbolic for any $a>1$ and parabolic when $a=1$. The same is true for $A_{a}^{m}$. Let us denote $C$ and $D$ in $G_{a}$ by $C_{a}$ and $D_{a}$. Since $\operatorname{tr}\left[C_{a}, D_{a}\right]$ is continuous on $a$, we have either $\operatorname{tr}\left[C_{a}, D_{a}\right] \leq-2$ for any $a \geq 1$ or $2 \leq \operatorname{tr}\left[C_{a}, D_{a}\right]$ for any $a \geq 1$.

If $\operatorname{tr}\left[C_{a}, D_{a}\right] \leq-2$, then

$$
J(C, D) \geq\left|\operatorname{tr}^{2} A_{a}-4\right|+|-2-2| \geq J\left(A_{a}, B\right) .
$$

If $2 \leq \operatorname{tr}\left[C_{a}, D_{a}\right]$, then $\lim _{b \rightarrow 1} \operatorname{tr}\left[C_{b}, D_{b}\right]=2$, and $J\left(C_{b}, D_{b}\right)$ is close to 0 when $b$ is close to 1 , which contradicts Jørgensen's inequality, because $G_{b}$ is non-elementary and discrete.

Case 2-2-3: $[C, D]$ is of type (iii). By Lemma 3.6, we have

$$
J(C, D)=\left|\operatorname{tr}^{2} C-4\right|+|\operatorname{tr}[C, D]-2| \geq\left|\operatorname{tr}^{2} A_{a}-4\right|+1=J\left(A_{a}, B\right) .
$$

Case 3: $C$ is of type (iii). By Lemma 3.6, we have

$$
J(C, D)=\left|\operatorname{tr}^{2} C-4\right|+|\operatorname{tr}[C, D]-2| \geq\left|\operatorname{tr}^{2} C-4\right| \geq 5 \geq J\left(A_{a}, B\right) .
$$

Hence, $J\left(G_{a}\right)=J\left(A_{a}, B\right)$ for $1 \leq a \leq a_{0}$, and the proposition is proved. 


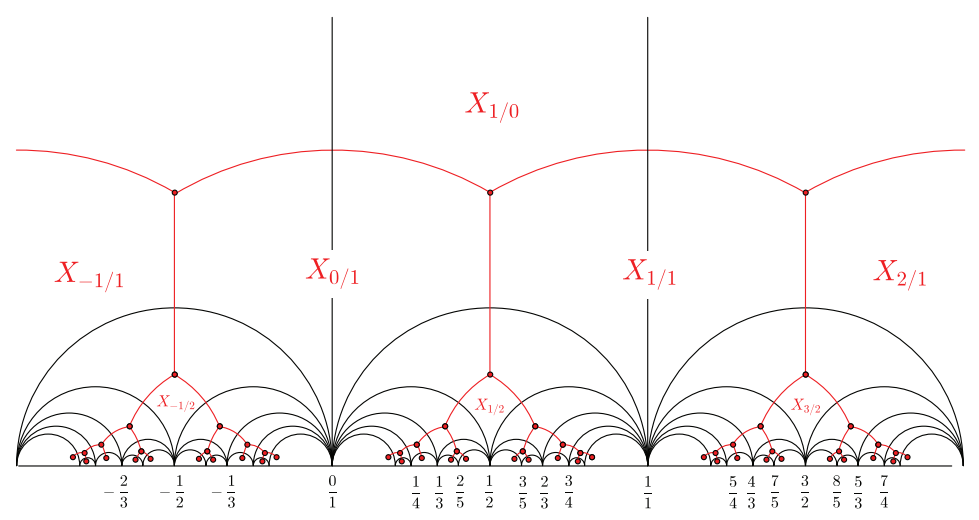

Figure 5. Farey triangulation and the dual graph $\Sigma$ (red).

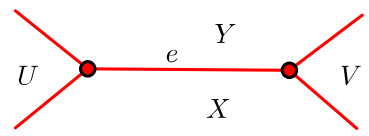

Figure $6 . e=(X, Y ; U, V)$.

\section{The Diagonal Slice of Schottky Space}

Jørgensen numbers can be studied by computer experiments. In this section, we present a computer generated picture (Figure (7) which estimates Jørgensen numbers in the diagonal slice of Schottky space.

4.1. Markoff maps. First, we recall Bowditch, Tan-Wong-Zhang theory [2,24] on Markoff maps very briefly. See section 3 in [24] for details.

We denote the set $\mathbb{Q} \cup\{1 / 0\}$ by $\widehat{\mathbb{Q}}$. Let $\mathcal{F}$ be the Farey triangulation of the upper half-plane $\mathbb{H}^{2}$. Recall that the vertex set of $\mathcal{F}$ is $\widehat{\mathbb{Q}}$, and two vertices $p / q$ and $r / s$ are connected in $\mathcal{F}$ by the hyperbolic geodesic connecting these two rational numbers if $p s-q r= \pm 1$. Let $\Sigma$ be the binary tree dual to $\mathcal{F}$. See Figure 5. A complementary region of $\Sigma$ is the closure of a connected component of the complement of $\Sigma$. The set of complementary regions of $\Sigma$ is denoted by $\Omega$. For each $X \in \Omega, \partial X \cap \mathbb{R}$ consists of a unique rational number, say $p / q$, and we denote this complementary region by $X_{p / q}$. See Figure 5 . Thus, we identify $\Omega$ with $\widehat{\mathbb{Q}}$. Let $e$ be an edge of $\Sigma$ with endpoints $u$ and $v$. Then, there exist $X, Y, U, V \in \Omega$ such that $e=X \cap Y$, $u=X \cap Y \cap U$, and $v=X \cap Y \cap V$. See Figure 6. We write $e=(X, Y ; U, V)$ to indicate these regions. A Markoff map is a map $\psi$ from $\Omega$ to $\mathbb{C}$ such that, for every edge $e=(X, Y ; U, V)$ in $\Sigma$, we have

$$
\psi(U)+\psi(V)=\psi(X) \psi(Y) .
$$

This condition is called the edge relation. Given $k \geq 0$, the set $\Omega_{\psi}(k)$ is defined by $\Omega_{\psi}(k)=\{X \in \Omega|| \psi(X) \mid \leq k\}$. We will need the following lemma. 
Lemma 4.1 (Theorem 3.1(2), 24]). Let $\psi$ be a Markoff map. For any $k \geq 2$, the union $\bigcup_{X \in \Omega_{\psi}(k)} X$ is connected as a subset of $\mathbb{H}^{2}$.

Let $F_{2}=\langle A, B\rangle$ be the free group on $A$ and $B$. An element $W$ of $F_{2}$ is called primitive if there exists an element $V$ such that $F_{2}=\langle W, V\rangle$, and $W$ and $V$ are called associated primitives. Let $\alpha$ be the abelianization homomorphism from $F_{2}$ onto $F_{2} / F_{2}^{\prime}\left(\cong \mathbb{Z}^{2}\right)$. Let $\rho$ be an $\operatorname{SL}(2, \mathbb{C})$ representation of $F_{2}$. Then, the next map $\psi_{\rho}: \Omega \rightarrow \mathbb{C}$ is well-defined:

$$
\psi_{\rho}\left(X_{n / m}\right)=\operatorname{tr} \rho\left(W_{n / m}\right),
$$

where $W_{n / m}$ is a primitive element such that $\alpha\left(W_{n / m}\right)=A^{m} B^{n}$. See Corollary 3.2 in [19. For example, we have

$$
\psi_{\rho}\left(X_{0 / 1}\right)=\operatorname{tr}_{\rho}(A), \quad \psi_{\rho}\left(X_{1 / 0}\right)=\operatorname{tr}_{\rho}(B), \quad \psi_{\rho}\left(X_{1 / 1}\right)=\operatorname{tr}_{\rho}(A B) .
$$

By Theorems 1.2 and 1.3 in [19] and the trace identity in $\operatorname{SL}(2, \mathbb{C})$,

$$
\operatorname{tr} A B+\operatorname{tr} A B^{-1}=\operatorname{tr} A \operatorname{tr} B,
$$

we have the next lemma.

Lemma 4.2. $\psi_{\rho}$ is a Markoff map.

4.2. The diagonal slice of Schottky space. Let us go back to the singular solid torus in section 2 . Recall that

$$
A_{a}=\left(\begin{array}{cc}
-3 a / 2 & 1 / 2 \\
-1 / 2 & -1 / 2 a
\end{array}\right), \quad B=\left(\begin{array}{cc}
i & 0 \\
0 & -i
\end{array}\right) .
$$

In this section, we consider that $A_{a}, B \in \mathrm{SL}(2, \mathbb{C})$. We denote $\left\langle A_{a}, B\right\rangle$ by $G_{a}$. Set

$$
x=2-\operatorname{tr} A_{a}^{2} .
$$

Then, we have

$$
\operatorname{tr} A_{a}=\sqrt{-x+2}, \quad \operatorname{tr} B=0, \quad \operatorname{tr} A_{a} B=\sqrt{x+1}, \quad \operatorname{tr}\left[A_{a}, B\right]=1 .
$$

See section 5.0.2 (in particular, Remark 5.4 for the sign of the square roots) in 23. From now on, we consider that $x$ is a complex parameter. The locus $\mathcal{D}$ in the $x$-plane of discrete and faithful representations was fully determined by computing Keen-Series pleating rays [23. (Here, "faithful" means that $\left\langle A_{a}, B\right\rangle$ is isomorphic to $\left\langle A_{1}, B\right\rangle$ as an abstract group.) $\mathcal{D}$ is called the diagonal slice of Schottky space. See Figure 7 The outside of the center black eye corresponds to $\mathcal{D}$. $\mathcal{D}$ is foliated by pleating rays. We will describe pleating rays briefly in the next subsection.

Let $a$ be a complex number such that $x=2-\operatorname{tr} A_{a}^{2} \in \mathcal{D}$. We denote the representation from $F_{2}=\langle\mathcal{A}, \mathcal{B}\rangle$ to $\operatorname{SL}(2, \mathbb{C})$ which sends $\mathcal{A}$ to $A_{a}$ and $\mathcal{B}$ to $B$ by $\rho_{x}$. Let $\psi_{x}: \widehat{\mathbb{Q}} \rightarrow \mathbb{C}$ be the Markoff map associated with $\left\langle A_{a}, B\right\rangle$ :

$$
\psi_{x}(0 / 1)=\operatorname{tr} A_{a}, \psi_{x}(1 / 0)=\operatorname{tr} B, \psi_{x}(1 / 1)=\operatorname{tr} A_{a} B .
$$

If $W$ and $V$ are associated primitives of $F_{2}$, then their images in $G_{a}$ generate $G_{a}$. By Lemma 2.1 and equation (요 $), \operatorname{tr} \rho_{x}([W, V])=\operatorname{tr}\left[A_{a}, B\right]=1$. Hence,

$$
J\left(\rho_{x}(W), \rho_{x}(V)\right)=\left|\psi_{x}^{2}(n / m)-4\right|+1 \geq J\left(G_{a}\right),
$$

where $n / m$ is determined by the abelianization $\alpha(W)=\mathcal{A}^{m} \mathcal{B}^{n}$. Hence,

$$
\Psi(x):=\inf _{q \in \widehat{\mathbb{Q}}}\left|\psi_{x}^{2}(q)-4\right|+1
$$




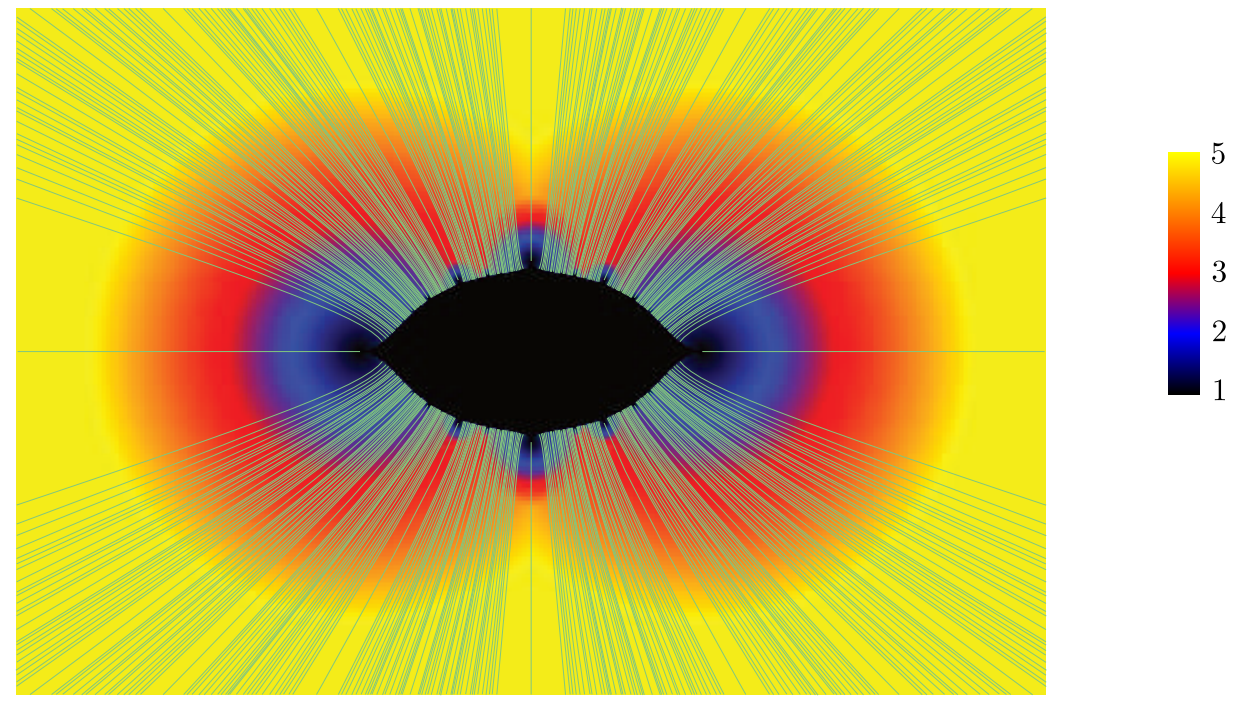

FiguRE 7. The diagonal slice and Jørgensen number: $-7 \leq \Re x \leq$ $8,-5 \leq \Im x \leq 5$. The color indicates the estimate of the Jørgensen number from above.

gives an estimate of $J\left(G_{a}\right)$ from above. We calculate $\Psi(x)$ by computer for $\{x \in$ $\mathbb{C} \mid-7 \leq \Re(x) \leq 8,-5 \leq \Im(x) \leq 5\}$. The colors outside the "black eye" in Figure 7 indicate the values of $\Psi(x)$. Since these groups are discrete and faithful (in particular non-elementary), $\Psi(x) \geq 1$. Since $\left|\psi_{x}^{2}(1 / 0)-4\right|+1=5$, we have $\Psi(x) \leq 5$. Figure 7 suggests that for each $1 \leq r \leq 5$, there are many non-elementary Kleinian groups in the diagonal slice with Jørgensen number less than or equal to $r$.

Remark 4.3. In practice, we cannot calculate $\psi_{x}^{2}(q)$ for all $q \in \widehat{\mathbb{Q}}$, and Figure 7 is an approximation of $\Psi(x)$ by calculating $\psi_{x}^{2}(q)$ for many $q \in \widehat{\mathbb{Q}}$. But, if $x$ is in the Bowditch set, in principle, we can calculate $\Psi(x)$. The key is Lemma 3.24 in [24]. The diagonal slice of Schottky space seems to coincide with the Bowditch set. See Section 2 in 23 .

Question 4.4. Does the equation $J\left(G_{a}\right)=\Psi(x)$ hold for each $x \in \mathcal{D}$ ? Note that, since $G_{a}$ is not free, there might be generating pairs of $G_{a}$ which do not come from associated primitives in $F_{2}$.

4.3. Jørgensen groups on the boundary of the diagonal slice. We consider the case $\Psi(x)=1$ on $\partial \mathcal{D}$. We begin by describing pleating rays very briefly. See 23 for details.

For $p / q \in \widehat{\mathbb{Q}}$, the real trace locus $\mathbb{R}_{p / q}$ of $p / q$ is $\left\{x \in \mathbb{C} \mid \psi_{x}(p / q) \in(-\infty,-2] \cup\right.$ $[2, \infty)\}$. The rational pleating ray $\mathcal{P}_{p / q}$ is a union of connected non-singular branches of $\mathbb{R}_{p / q}$ (Corollary 4.11, [23]). The rational pleating rays are indexed by $\widehat{\mathbb{Q}} / \sim$, where $p / q \sim p^{\prime} / q^{\prime}$ if and only if $p^{\prime} / q^{\prime}= \pm p / q+2 k, k \in \mathbb{Z}$ (Proposition 4.8, [23]). (If $p / q \sim p^{\prime} / q^{\prime}$, then we have $\psi_{x}(p / q)= \pm \psi_{x}\left(p^{\prime} / q^{\prime}\right)$.) 
If $p / q$ is not an integer, $\mathcal{P}_{p / q}$ consists of two components (branches). One is in the upper half-plane, and the other in the lower half-plane. Let $e_{p / q}$ be the endpoint of $\mathcal{P}_{p / q}$ in the upperhalf-plane, or on the real axis if $p / q$ is an integer. By construction, we have $\psi_{e_{p / q}}^{2}(p / q)-4=0$. Hence, $\Psi\left(e_{p / q}\right)=1$, and the corresponding representation is a Jørgensen group of parabolic type. (If $\langle A, B\rangle$ is a Jørgensen group with $J(A, B)=1$ and $A$ parabolic, it is called a Jørgensen group of parabolic type [13.)

In order to describe these groups, we recall the following Li-Oichi-Sato normalization.

Lemma 4.5 (Lemma 3.1, 13). Let $M$ and $N$ be elements of $\operatorname{PSL}(2, \mathbb{C})$ such that $M$ is parabolic and $N$ is elliptic or loxodromic. Then, $M, N$ can be normalized as

$$
M=\left(\begin{array}{ll}
1 & 1 \\
0 & 1
\end{array}\right), \quad N_{\sigma, \mu}=\left(\begin{array}{cc}
\mu \sigma & \mu^{2} \sigma-1 / \sigma \\
\sigma & \mu \sigma
\end{array}\right),
$$

where $\sigma \in \mathbb{C} \backslash\{0\}$ and $\mu \in \mathbb{C}$.

Remark 4.6. Li-Oichi-Sato [13, [12, 14] considered the case $\mu=i k, k \in \mathbb{R}$. They conjectured that, for any Jørgensen group $G$ of parabolic type, there exists a marked group $G_{\sigma, i k}=\left\langle M, N_{\sigma, i k}\right\rangle, \sigma \in \mathbb{C} \backslash\{0\}, k \in \mathbb{R}$, such that $G_{\sigma, i k}$ is conjugate to $G$. Later, Callahan [3] found counterexamples for this conjecture.

Now, we compute some examples. We begin by calculating some Markoff maps and the endpoints of pleating rays:

$$
\begin{array}{ll}
\psi_{x}^{2}(0 / 1)-4=(-x+2)-4, & e_{0 / 1}=-2, \\
\psi_{x}^{2}(1 / 3)-4=(-x+1)^{2}(x+1)-4, & e_{1 / 3} \approx-0.5652+1.0434 i, \\
\psi_{x}^{2}(3 / 8)-4=(-x+2)(x+1)\left(x^{3}-x^{2}-1\right)^{2}-4, & e_{3 / 8} \approx-0.2992+1.0726 i, \\
\psi_{x}^{2}(2 / 5)-4=x^{4}(-x+2)-4, & e_{2 / 5} \approx-0.1372+1.1260 i, \\
\psi_{x}^{2}(1 / 2)-4=(-x+2)(x+1)-4, & e_{1 / 2}=(1+\sqrt{7} i) / 2 .
\end{array}
$$

(Note that the equation $\psi_{x}^{2}(p / q)=4$ has many roots, and in order to make the right choice for $e_{p / q}$, we need [23.) Let $A_{p / q}$ denote the matrix in (6) such that $e_{p / q}=2-\operatorname{tr} A_{a}^{2}$. Then, for example,

$$
\begin{aligned}
& \left(A_{0 / 1}, B\right),\left(A_{1 / 3}^{3} B, A_{1 / 3}\right),\left(A_{3 / 8}^{3} B A_{3 / 8}^{3} B A_{3 / 8}^{2} B, A_{3 / 8}^{3} B\right), \\
& \left(A_{2 / 5}^{3} B A_{2 / 5}^{2} B, A_{2 / 5}^{3} B\right),\left(A_{1 / 2}^{2} B, A_{1 / 2}\right)
\end{aligned}
$$

generate Jørgensen groups of parabolic type. The Li-Oichi-Sato parameters for these groups are as follows:

$$
\begin{aligned}
& (\sigma, \mu)=(i, 0) \\
& \text { for }\left(A_{0 / 1}, B\right) \text {, } \\
& (\sigma, \mu) \approx(-i, 0.1597+0.8166 i) \\
& \text { for }\left(A_{1 / 3}^{2} B, A_{1 / 3}\right) \text {, } \\
& (\sigma, \mu) \approx(i, 0.1839+0.9356 i) \\
& \text { for }\left(A_{3 / 8}^{3} B A_{3 / 8}^{3} B A_{3 / 8}^{2} B, A_{3 / 8}^{3} B\right) \text {, } \\
& (\sigma, \mu) \approx(i, 0.3016+0.9041 i) \\
& \text { for }\left(A_{2 / 5}^{3} B A_{2 / 5}^{2} B, A_{2 / 5}^{3} B\right) \text {, } \\
& (\sigma, \mu)=(i,(1+\sqrt{7} i) / 4) \\
& \text { for }\left(A_{1 / 2}^{2} B, A_{1 / 2}\right) \text {. }
\end{aligned}
$$

We conjecture that groups which correspond to the endpoints of the rational pleating rays (except -2 and 3 on the real axis) are counterexamples for Li-Oichi-Sato's conjecture in Remark 4.6 . 
Remark 4.7. The limit set of $\left\langle M, N_{\sigma, \mu}\right\rangle$ with $(\sigma, \mu)=(i, 0)$ is the real line. The limit sets of other examples are complicated and beautiful. For example, see Figure 8 for the limit set of the third case $(\sigma, \mu) \approx(i, 0.1839+0.9356 i)$.

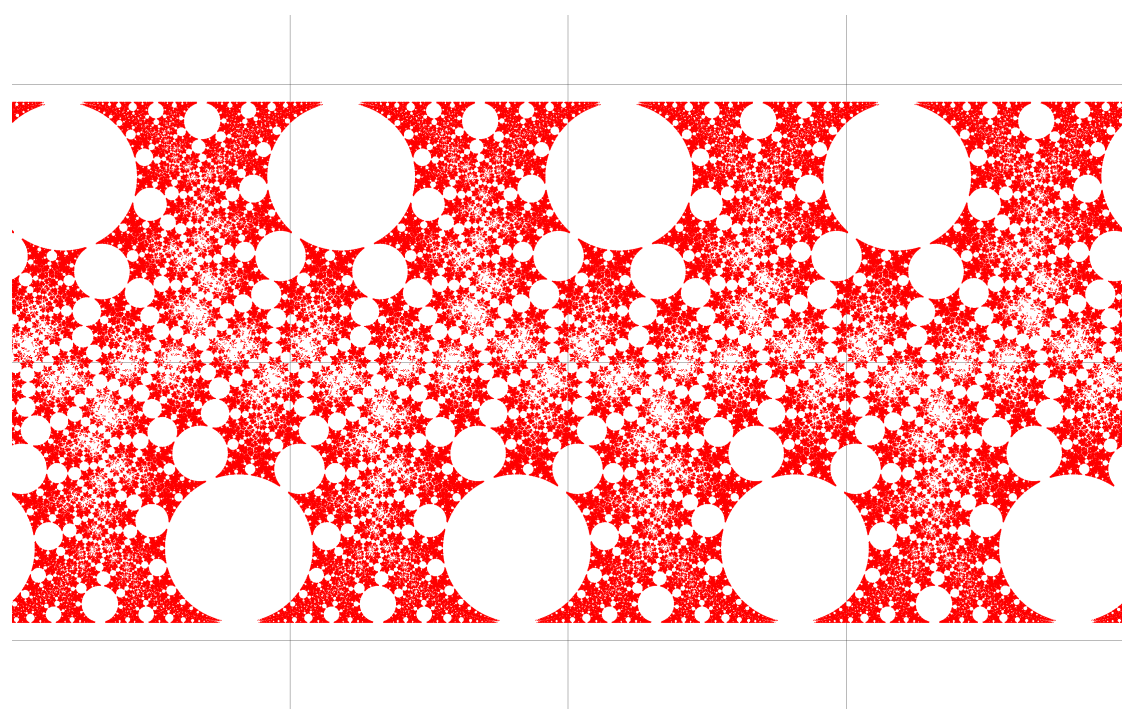

FiguRE 8. The limit set of $\left\langle M, N_{\sigma, \mu}\right\rangle$ with $(\sigma, \mu) \approx(i, 0.1839+0.9356 i)$.

\section{ACKNOWLEDGMENTS}

The authors are grateful to Hiroki Sato for his valuable comments and suggestions on the early version of this paper. The authors would like to thank Yohei Komori and Takuya Sakasai for their helpful advice and encouragement. Finally, the authors thank the anonymous referee for his/her careful reading of our paper, which significantly contributed to improving the quality of the paper.

\section{REFERENCES}

[1] Alan F. Beardon, The geometry of discrete groups, Graduate Texts in Mathematics, vol. 91, Springer-Verlag, New York, 1983. MR698777

[2] B. H. Bowditch, Markoff triples and quasi-Fuchsian groups, Proc. London Math. Soc. (3) 77 (1998), no. 3, 697-736, DOI 10.1112/S0024611598000604. MR1643429

[3] Jason Callahan, Jørgensen number and arithmeticity, Conform. Geom. Dyn. 13 (2009), 160186, DOI 10.1090/S1088-4173-09-00196-9. MR2525101

[4] Werner Fenchel, Elementary geometry in hyperbolic space, De Gruyter Studies in Mathematics, vol. 11, Walter de Gruyter \& Co., Berlin, 1989. With an editorial by Heinz Bauer. MR.1004006

[5] F. W. Gehring and G. J. Martin, Stability and extremality in Jørgensen's inequality, Complex Variables Theory Appl. 12 (1989), no. 1-4, 277-282, DOI 10.1080/17476938908814372. MR.1040927

[6] Francisco González-Acuña and Arturo Ramírez, Jørgensen subgroups of the Picard group, Osaka J. Math. 44 (2007), no. 2, 471-482. MR2351012

[7] Troels Jørgensen, On discrete groups of Möbius transformations, Amer. J. Math. 98 (1976), no. 3, 739-749, DOI 10.2307/2373814. MR0427627

[8] Troels Jørgensen and Maire Kiikka, Some extreme discrete groups, Ann. Acad. Sci. Fenn. Ser. A I Math. 1 (1975), no. 2, 245-248. MR0399452 
[9] Linda Keen and Caroline Series, Pleating coordinates for the Maskit embedding of the Teichmüller space of punctured tori, Topology 32 (1993), no. 4, 719-749, DOI 10.1016/00409383(93)90048-Z. MR.1241870

[10] Linda Keen and Caroline Series, The Riley slice of Schottky space, Proc. London Math. Soc. (3) 69 (1994), no. 1, 72-90, DOI 10.1112/plms/s3-69.1.72. MR.1272421

[11] Yohei Komori and Caroline Series, The Riley slice revisited, The Epstein birthday schrift, Geom. Topol. Monogr., vol. 1, Geom. Topol. Publ., Coventry, 1998, pp. 303-316, DOI 10.2140/gtm.1998.1.303. MR.1668296

[12] Changjun Li, Makito Oichi, and Hiroki Sato, Jørgensen groups of parabolic type II (countably infinite case), Osaka J. Math. 41 (2004), no. 3, 491-506. MR2107659

[13] Changjun Li, Makito Oichi, and Hiroki Sato, Jørgensen groups of parabolic type I (finite case), Comput. Methods Funct. Theory 5 (2005), no. 2, 409-430, DOI 10.1007/BF03321107. MR2205423

[14] Changjun Li, Makito Oichi, and Hiroki Sato, Jørgensen groups of parabolic type III (uncountably infinite case), Kodai Math. J. 28 (2005), no. 2, 248-264. MR2153913

[15] Wilhelm Magnus, Abraham Karrass, and Donald Solitar, Combinatorial group theory: Presentations of groups in terms of generators and relations, Second revised edition, Dover Publications, Inc., New York, 1976. MR0422434

[16] David Mumford, Caroline Series, and David Wright, Indra's pearls: The vision of Felix Klein, Cambridge University Press, New York, 2002. MR1913879

[17] Makito Oichi, A fundamental polyhedron for the figure-eight knot group, Topology Appl. 146/147 (2005), 15-19, DOI 10.1016/j.topol.2002.10.001. MR2107132

[18] Makito Oichi and Hiroki Sato, Jørgensen numbers of discrete groups, Sūrikaisekikenkyūsho Kōkyūroku 1519 (2006), 105-118.

[19] R. P. Osborne and H. Zieschang, Primitives in the free group on two generators, Invent. Math. 63 (1981), no. 1, 17-24, DOI 10.1007/BF01389191. MR608526

[20] Hiroki Sato, One-parameter families of extreme discrete groups for Jørgensen's inequality, In the tradition of Ahlfors and Bers (Stony Brook, NY, 1998), Contemp. Math., vol. 256, Amer. Math. Soc., Providence, RI, 2000, pp. 271-287, DOI 10.1090/conm/256/04013. MR1759686

[21] Hiroki Sato, The Picard group, the Whitehead link and Jørgensen groups, Progress in analysis, Vol. I, II (Berlin, 2001), World Sci. Publ., River Edge, NJ, 2003, pp. 149-158. MR2032679

[22] Hiroki Sato, The Jørgensen number of the Whitehead link group, Bol. Soc. Mat. Mexicana (3) 10 (2004), Special Issue, 495-502. MR2199365

[23] Caroline Series, Ser Peow Tan, and Yasushi Yamashita, The diagonal slice of Schottky space, Algebr. Geom. Topol. 17 (2017), no. 4, 2239-2282, DOI 10.2140/agt.2017.17.2239. MR 3685607

[24] Ser Peow Tan, Yan Loi Wong, and Ying Zhang, Generalized Markoff maps and McShane's identity, Adv. Math. 217 (2008), no. 2, 761-813, DOI 10.1016/j.aim.2007.09.004. MR2370281

[25] A. Yu. Vesnin and A. V. Maslě̆, On Jørgensen numbers and their analogs for groups of figure-eight orbifolds (Russian, with Russian summary), Sibirsk. Mat. Zh. 55 (2014), no. 5, 989-1000, DOI 10.1134/s0037446614050036; English transl., Sib. Math. J. 55 (2014), no. 5, 807-816. MR3289108

[26] Ryosuke Yamazaki, Some extensions of Oichi-Sato's theorem for the Jørgensen numbers of the Kleinian groups, 2016, Master thesis, University of Tokyo.

NAra Women's University, Kitauoyanishi-Machi, NARA-Shi, NARA 630-8506, Japan

Email address: yamasita@ics.nara-wu.ac.jp

Gakushuin Boys' Senior High School, 1-5-1 Mejiro, Toshima-ku, Tokyo 171-0031, Japan

Email address: rsk.yamazaki.ms@gmail.com 